\title{
Anomalous magnetoresistance oscillations and enhanced superconductivity in single-crystal $\mathrm{Pb}$ nanobelts
}

\author{
Jian Wang, ${ }^{1}$ Xu-Cun Ma, ${ }^{2} \mathrm{Li} \mathrm{Lu}^{2}{ }^{2} \mathrm{Ai}-\mathrm{Zi} \mathrm{Jin},{ }^{2}$ Chang-Zhi Gu,${ }^{2}$ X. C. Xie, ${ }^{3} \mathrm{Jin}-F e n g ~ J i a,{ }^{4}$ \\ Xi Chen, ${ }^{4}$ and Qi-Kun Xue ${ }^{4, a)}$ \\ ${ }_{1}^{1}$ Institute of Physics, Chinese Academy of Sciences, Beijing 100080, China and Center for Nanoscale \\ Science and Department of Physics, Pennsylvania State University, University Park, \\ Pennsylvania 16802-6300, USA \\ ${ }^{2}$ Institute of Physics, Chinese Academy of Sciences, Beijing 100080, China \\ ${ }^{3}$ Institute of Physics, Chinese Academy of Sciences, Beijing 100080, China and Department of Physics, \\ Oklahoma State University, Stillwater, Oklahoma 74078, USA \\ ${ }^{4}$ Department of Physics, Tsinghua University, Beijing 100084, China
}

(Received 27 March 2008; accepted 20 May 2008; published online 13 June 2008)

\begin{abstract}
Using atomically flat single-crystal $\mathrm{Pb}$ ultrathin films grown on $\mathrm{Si}(111)$ substrate by molecular beam epitaxy, we fabricated $\mathrm{Pb}$ nanobelts of 28 atomic monolayers thick, $285 \mathrm{~nm}$ wide, and $10 \mu \mathrm{m}$ long by focus ion beam technique. The transport property is measured and the results are compared to the $\mathrm{Pb}$ films. We find that below the superconducting transition temperature $T_{C}$, the resistance of the nanobelts exhibits clear and reproducible oscillations as a function of perpendicular magnetic field, which are not observed in the $\mathrm{Pb}$ films. In addition, $T_{C}$ enhancement was also observed in the nanobelts. The results suggest that the single-crystal $\mathrm{Pb}$ nanobelts prepared on $\mathrm{Si}$ semiconductor substrate offer a great opportunity for integrating superconducting circuits in a single Si chip.

(C) 2008 American Institute of Physics. [DOI: 10.1063/1.2945280]
\end{abstract}

Superconductivity in quasi-one-dimensional (quasi-1D) nanowires is one of the most intriguing problems in condensed-matter physics. ${ }^{1-6}$ Many interesting physical phenomena, such as thermally activated phase slip, ${ }^{7-9}$ quantum phase slip, ${ }^{10,11}$ superconductor-insulator transition, ${ }^{12}$ and proximity effect, ${ }^{13,14}$ were observed in quasi-1D systems as lately developed experimental techniques have enabled the fabrication of superconducting wires with a diameter reaching to certain degree of the 1D limit. ${ }^{15,16}$ Magnetic field lifts the time reversal symmetry of the spin and orbital states of paired electrons and suppresses the superconducting transition; ${ }^{17}$ many interesting results such as magnetoresistance oscillations were also observed under a magnetic field. ${ }^{7,18-20}$ However, the nanowire samples used in these studies were either amorphous or granular, or their sizes are ill defined. To understand these interesting behaviors, singlecrystal superconducting systems with controlled dimensionality are highly desirable.

Here, we report on a method to fabricate single-crystal $\mathrm{Pb}$ nanobelts with nanometer scale control of their sizes by using combined molecular beam epitaxy (MBE) thin film growth and focus ion beam etching techniques. These $\mathrm{Pb}$ nanobelts exhibit enhanced $T_{C}$ and reproducible resistance oscillations as a function of magnetic field perpendicular to the plane of the sample below $T_{C}$, which were not found in the $\mathrm{Pb}$ films of the same thickness from which the nanobelts were made, and are also different from previous reports. ${ }^{18,20}$

The experiments were performed in an ultrahigh vacuum low-temperature scanning tunneling microscopy (STM) system with a MBE chamber for in situ preparation of thin films (Unisoku USM1300). The Pb thin films used for fabrication of nanobelts were prepared on high resistivity $\mathrm{Si}(111)$ substrates, which are insulating at low temperature. ${ }^{21}$ The details were reported elsewhere. ${ }^{22-27}$ Figure 1(a) shows a typical

a)Electronic mail: qkxue@mail.tsinghua.edu.cn.
STM image of the atomically smooth $\mathrm{Pb}$ films with a thickness of 28 atomic monolayers (MLs). To fabricate nanobelts, the film was deposited with $4 \mathrm{ML} \mathrm{Au}$ as a protection cover ${ }^{22}$ and was transferred out of the UHV growth chamber. The

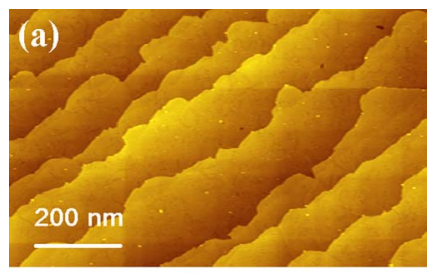

(b)
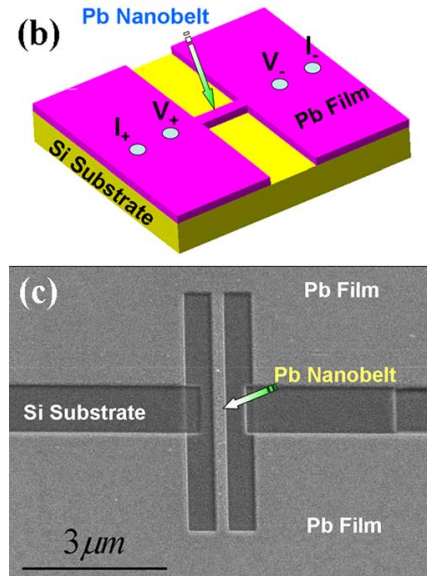

FIG. 1. (Color online) (a) A scanning tunneling microscope image of the 28 ML atomically smooth $\mathrm{Pb}$ thin film on the $\mathrm{Si}(111) 7 \times 7$ substrate. The image was recorded at $80 \mathrm{~K}$ with a constant current of $100 \mathrm{pA}$. (b) The schematic graph for the transport measurement across the $\mathrm{Pb}$ nanobelt. The four contact electrodes are made of indium. (c) A scanning electron micrograph (SEM) of the $\mathrm{Pb}$ nanobelt made of the $\mathrm{Pb}$ film shown in (a). The $\mathrm{Pb}$ nanobelt is $28 \mathrm{ML}$ thick, $285 \mathrm{~nm}$ wide and $10 \mu \mathrm{m}$ long. The dark region on the two sides of the $\mathrm{Pb}$ nanobelt is exposed $\mathrm{Si}$ surface, which isolates two blocks of the $\mathrm{Pb}$ film. The dimension in the length direction of the nanobelt is compressed since the SEM picture was taken at the $52^{\circ}$ tilted sample stage in a FIB system. 

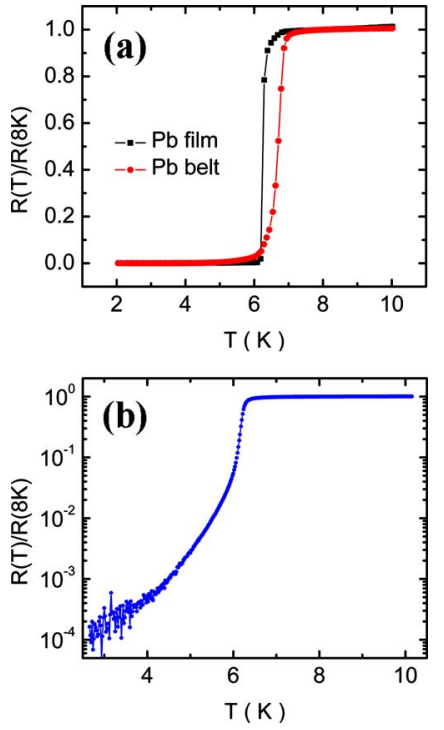

FIG. 2. (Color online) (a) Resistance as a function of temperature measured from the $\mathrm{Pb}$ film in Fig. 1(a) and the Pb nanobelt in Fig. 1(c), respectively. The vertical scale is normalized to the resistance at $T=8 \mathrm{~K}$. The transition temperature $T_{C}$ of the $\mathrm{Pb}$ nanobelt is obvious higher than the $T_{C}$ of the $\mathrm{Pb}$ film. (b) Logarithm resistance vs temperature of a $300 \mathrm{~nm}$ wide and $23 \mathrm{ML}$ thick $\mathrm{Pb}$ nanobelt. Below $T_{C}$, the resistance tail is obvious.

nanobelts were then made by a commercial focused ion $(\mathrm{Ga})$ beam etching system (FEI-DB235), as schematically shown in the Fig. 1(b). The etching current was set less than $10 \mathrm{pA}$ to minimize contamination and structure damage by the $\mathrm{Ga}$ ions. Four indium electrodes with Au wires of $25 \mu \mathrm{m}$ in diameter were made and connected to the surface of the two parts of the film [see Fig. 1(b)] for transport measurements. Since the thickness of the $\mathrm{Pb}$ films can be precisely controlled, the nanobelt shown in Fig. 1(c) exhibits well-defined dimensionality and is of single crystal except for trivial damage of its long edge sides by the ions, as compared to the amorphous and granular samples previously studied. ${ }^{15,18,20}$

The $\mathrm{Pb}$ nanobelt shown in Fig. 1(c) is $28 \mathrm{ML}(\sim 8 \mathrm{~nm})$ thick, $285 \mathrm{~nm}$ wide, and $10 \mu \mathrm{m}$ long, and the equivalent cross section is about $48 \times 48 \mathrm{~nm}^{2}$, which is comparable to the BCS coherent length of bulk $\mathrm{Pb}$ at $0 \mathrm{~K}, \xi_{0}^{\text {bulk }}=90.5 \mathrm{~nm}$ (Ref. 28) and the coherence length of $\sim 27 \mathrm{~nm}$ in the $\mathrm{Pb}$ film [estimated by $\xi \approx\left(\xi_{0} l\right)^{1 / 2}$ at the dirty limit, ${ }^{25}$ where $l$ is the mean free path for the film]. Please note that the $\mathrm{Pb}$ nanobelt is $285 \mathrm{~nm}$ wide, while the size of the vortex core (which is the same as the coherence length) in the $\mathrm{Pb}$ film is only $27 \mathrm{~nm}$. Thus vortices can enter into the belt and the $\mathrm{Pb}$ nanobelt is not $1 \mathrm{D}$ or quasi-1D superconductor. ${ }^{29}$ However, with a very small thickness-to-width ratio $(<0.03)$ the singlecrystal $\mathrm{Pb}$ nanobelt shows different behavior with the $\mathrm{Pb}$ film and displays some characteristics of 1D superconductor, as discussed below. 1D behavior in quasi two-dimensional samples was reported in disordered superconducting $\mathrm{NbN}$ nanowires and wide superconducting strips with a hole in the middle. $^{30,31}$

Figure 2(a) shows $R$ - $T$ curves of the $28 \mathrm{ML} \mathrm{Pb}$ film [see Fig. 1(a)] and the $\mathrm{Pb}$ nanobelt [see Fig. 1(c) for comparison]. Both the film and nanobelt show nearly ideal superconducting transition and almost zero residual resistance, indicating high quality of the samples. We also note that the onset $T_{C}$ $(6.9 \mathrm{~K})$ of the $\mathrm{Pb}$ nanobelt is obviously higher than that $(6.3 \mathrm{~K})$ of the $\mathrm{Pb}$ film. The enhancement of $T_{C}$ was verified Author complimentary copy. Redistribution subject to AIP
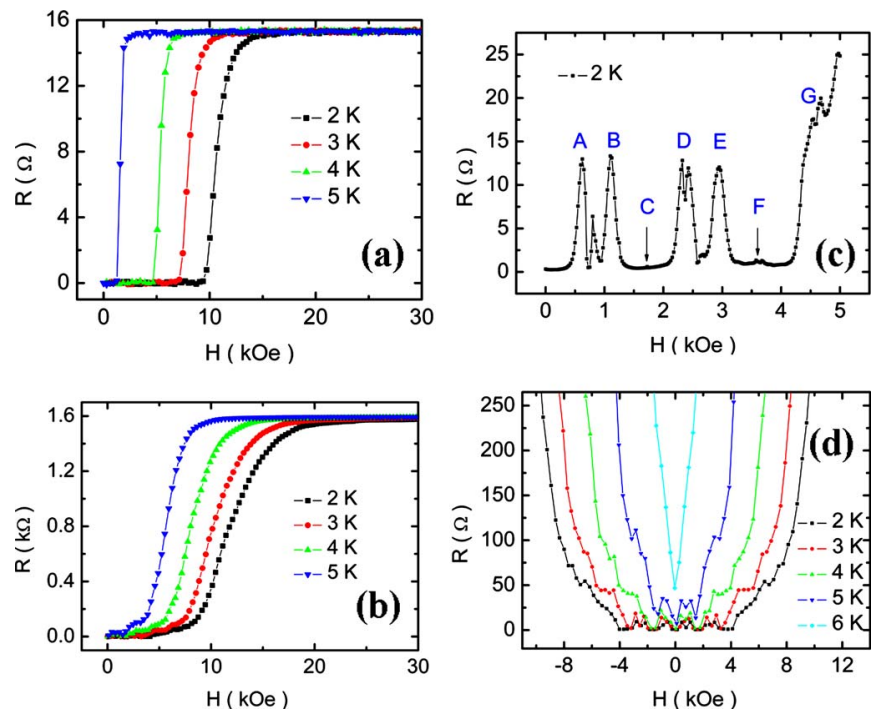

FIG. 3. (Color online) (a) Magnetoresistance of the Pb film in Fig. 1(a) with a magnetic field applied perpendicular to the film at different temperatures. (b) Magnetoresistance of the $\mathrm{Pb}$ nanobelt in Fig. 1(c) with a magnetic field applied perpendicular to the film at different temperatures. (c) Close view of (b) in low magnetic field regime at $2 \mathrm{~K}$ for clarity. (d) Magnetoresistance oscillations of the $\mathrm{Pb}$ nanobelt in Fig. 1(c) at 2, 3, 4, 5 and $6 \mathrm{~K}$, respectively, with both positive and negative perpendicular fields.

in another three samples. We know that the $T_{C}$ of the ultrathin single-crystal $\mathrm{Pb}$ film is lower than the $T_{C}$ of the bulk $\mathrm{Pb}^{22-27,32}$ However, from $\mathrm{Pb}$ film to $\mathrm{Pb}$ nanobelt, the $T_{C}$ is increased.

One may think that the enhancement of $T_{C}$ is related to the enhanced surface electron-phonon scattering. ${ }^{33,34}$ However, the surface area in the nanobelt is increased only by $0.2 \%$ compared to the thin film case, which is not enough to explain the increase of $9 \%$. Currently, we have no satisfied explanation of this observation.

In order to study the $R-T$ property of the $\mathrm{Pb}$ nanobelt at low temperature, the logarithm of the resistance versus temperature for a $300 \mathrm{~nm}$ wide and $23 \mathrm{ML}$ thick $\mathrm{Pb}$ nanobelt is plotted in Fig. 2(b). We can see clear nonlinear resistance tail below $T_{C}$. The curve is smooth and shows a positive curvature, as one expect from the models with quantum phase slips in superconducting nanowires. ${ }^{10}$ The behavior was confirmed in other samples.

Figure 3(a) shows the resistance of the $28 \mathrm{ML}$ film as a function of the magnetic field $(H)$ applied perpendicularly to the film at different temperatures (below $T_{C}$ ). The film shows a sharp superconducting transition and almost zero residual resistance. We can see that below $T_{C}$ the upper critical field $H_{C \perp}$ of the superconducting $\mathrm{Pb}$ film gradually decreases with increasing temperature. Figure 3(b) shows the corresponding $R-H$ curves of the $\mathrm{Pb}$ nanobelt. The critical field $H_{C \perp}$ of the superconducting nanobelt gradually decreases with temperature; however, the superconducting transition region becomes wider. It is noted that the normal state resistance of the $\mathrm{Pb}$ film is $\sim 15 \Omega$ and the $\mathrm{Pb}$ nanobelt is $\sim 1.6 \mathrm{k} \Omega$ in the normal state. The $\mathrm{Pb}$ film is about $2 \mathrm{~mm}$ wide and the distance between the two voltage electrodes is about $0.7 \mathrm{~mm}$. According to the film resistance $(15 \Omega)$, we can derive that the resistance of the nanobelt should be about $1.5 \mathrm{k} \Omega$, which is close to our measurement result $(1.6 \mathrm{k} \Omega)$. Furthermore, below the $H_{C \perp}$ of the $\mathrm{Pb}$ nanobelt, the magnetoresistance exhibits some oscillations in superconducting state. 
To further understand the magnetoresistance behavior of the superconducting $\mathrm{Pb}$ nanobelt, we show the $R-H$ curve of the $\mathrm{Pb}$ nanobelt in low magnetic field regime at $2 \mathrm{~K}$ (below $\left.T_{C}\right)$ in Fig. 3(c). An oscillation in magnetic resistance is evident. At zero field, the resistance is close to zero (less than $1 \Omega$ ). With increasing field, the resistance remains small and reaches peak "A" at $0.6 \mathrm{kOe}$ at which another resistance of $13 \Omega$ appears. With further increasing field, the resistance oscillates. From the "A" to "F," we can see an oscillating period of 0.6 kOe although the "C" and F are suppressed and the periodicity is not well defined. In addition, from the " $\mathrm{D}$," "F," and "G" double peaks are present. Due to the transition at $H_{C \perp}$, the "G" deviates the oscillation period. Above $5 \mathrm{kOe}$, the resistance increases continually and the oscillations disappear gradually. Figure 3(d) shows magnetoresistance oscillations at various temperatures below $T_{C}$ in both positive and negative perpendicular fields. This figure gives evidence that the oscillation in the $\mathrm{Pb}$ nanobelt is reproducible (not just some sort of noise) and might be considered quasiperiodic. The big oscillation amplitude of more than $10 \Omega$ can be exploited to fabricate nanoscale SQUID-like ${ }^{18}$ devices and on-off functional and logical nanodevices.

The observed magnetoresistance oscillation is not understood well currently. It seems that there are mesoscopic superconducting ringlike structures in the $\mathrm{Pb}$ nanobelt, which might induce the periodic magnetoresistance oscillation in superconducting regime, such as Little-Parks-like ${ }^{35}$ oscillations. We note that although the oscillation is reproducible for different samples, the period of oscillation is not well defined. A possible explanation is that some normal regions might occur in the nanobelt or in the films connected to the nanobelt in the presence of a magnetic field. These regions can block the current and cause a random resistance fluctuation, as reported previously by Prozorov et al. ${ }^{36}$ In this case, the magnetoresistance oscillations cannot be periodic.

This work was financially supported by National Science Foundation and Ministry of Science and Technology of China.

\footnotetext{
${ }^{1}$ A. V. Herzog, P. Xiong, F. Sharifi, and R. C. Dynes, Phys. Rev. Lett. 76, 668 (1996)

${ }^{2}$ B. Camarota, F. Parage, P. Delsing, and O. Buisson, Phys. Rev. Lett. 86, 480 (2001)

${ }^{3}$ D. Y. Vodolazov, F. M. Peeters, L. Piraux, S. Mátéfi-Tempfli, and S. Michotte, Phys. Rev. Lett. 91, 157001 (2003).

${ }^{4}$ M. L. Tian, N. Kumar, S. Y. Xu, J. G. Wang, J. S. Kurtz, and M. H. W.
}

Chan, Phys. Rev. Lett. 95, 076802 (2005).

${ }^{5}$ M. Zgirski, K.-P. Riikonen, V. Touboltsev, and K. Arutyunov, Nano Lett. 5, 1029 (2005).

${ }^{6}$ F. Altomare, A. M. Chang, M. R. Melloch, Y. Hong, and C. W. Tu, Phys. Rev. Lett. 97, 017001 (2006).

${ }^{7}$ A. Rogachev, A. T. Bollinger, and A. Bezryadin, Phys. Rev. Lett. 94, 017004 (2005).

${ }^{8}$ J. E. Lukens, R. J. Warburton, and W. W. Webb, Phys. Rev. Lett. 25, 1180 (1970).

${ }^{9}$ W. A. Little, Phys. Rev. 156, 396 (1967).

${ }^{10}$ C. N. Lau, N. Markovic, M. Bockrath, A. Bezryadin, and M. Tinkham, Phys. Rev. Lett. 87, 217003 (2001).

${ }^{11}$ N. Giordano, Phys. Rev. Lett. 61, 2137 (1988).

${ }^{12}$ F. Sharifi, A. V. Herzog, and R. C. Dynes, Phys. Rev. Lett. 71, 428 (1993).

${ }^{13}$ G. R. Boogaard, A. H. Verbruggen, W. Belzig, and T. M. Klapwijk, Phys. Rev. B 69, 220503 (2004).

${ }^{14}$ G. E. Blonder, M. Tinkham, and T. M. Klapwijk, Phys. Rev. B 25, 4515 (1982).

${ }^{15}$ A. Bezryadin, C. N. Lau, and M. Tinkham, Nature (London) 404, 971 (2000).

${ }^{16}$ M. Remeika and A. Bezryadin, Nanotechnology 16, 1172 (2005).

${ }^{17}$ K. Maki and P. Fulde, Phys. Rev. 140, A1586 (1965).

${ }^{18}$ A. Johansson, G. Sambandamurthy, D. Shahar, N. Jacobson, and R. Tenne, Phys. Rev. Lett. 95, 116805 (2005).

${ }^{19}$ P. Xiong, A. V. Herzog, and R. C. Dynes, Phys. Rev. Lett. 78, 927 (1997).

${ }^{20}$ A. V. Herzog, P. Xiong, and R. C. Dynes, Phys. Rev. B 58, 14199 (1998).

${ }^{21}$ J. L. Li, J. F. Jia, X. J. Liang, X. Liu, J. Z. Wang, Q. K. Xue, Z. Q. Li, J. S. Tse, Z. Y. Zhang, and S. B. Zhang, Phys. Rev. Lett. 88, 066101 (2002).

${ }^{22}$ Y. Guo, Y. F. Zhang, X. Y. Bao, T. Z. Han, Z. Tang, L. X. Zhang, W. G. Zhu, E. G. Wang, Q. Niu, Z. Q. Qiu, J. F. Jia, Z. X. Zhao, and Q. K. Xue, Science 306, 1915 (2004).

${ }^{23}$ T.-C. Chiang, Science 306, 1900 (2004).

${ }^{24}$ Y. F. Zhang, J. F. Jia, T. Z. Han, Z. Tang, Q. T. Shen, Y. Guo, Z. Q. Qiu, and Q. K. Xue, Phys. Rev. Lett. 95, 096802 (2005).

${ }^{25}$ X. Y. Bao, Y. F. Zhang, Y. P. Wang, J. F. Jia, Q. K. Xue, X. C. Xie, and Z. X. Zhao, Phys. Rev. Lett. 95, 247005 (2005).

${ }^{26}$ M. M. Ozer, J. R. Thompson, and H. H. Weitering, Nat. Phys. 2, 173 (2006).

${ }^{27}$ J. Wang, X. C. Ma, Y. Qi, Y. S. Fu, S. H. Ji, L. Lu, J. F. Jia, and Q. K. Xue, Appl. Phys. Lett. 90, 113109 (2007).

${ }^{28}$ Mustafa M. Özer, J. R. Thompson, and H. H. Weitering, Phys. Rev. B 74, 235427 (2006).

${ }^{29}$ K. K. Likharev, Rev. Mod. Phys. 51, 101 (1979).

${ }^{30}$ M. Bell, N. Kaurova, A. Divochiy, G. Gol'tsman, J. Bird, A. Sergeev, and A. Verevkin, IEEE Trans. Appl. Supercond. 17, 267 (2007).

${ }^{31}$ A. G. Sivakov, A. M. Glukhov, A. N. Omelyanchouk, Y. Koval, P. Müller, and A. V. Ustinov, Phys. Rev. Lett. 91, 267001 (2003).

${ }^{32}$ D. Eom, S. Qin, M.-Y. Chou, and C. K. Shih, Phys. Rev. Lett. 96, 027005 (2006).

${ }^{33}$ M. Tian, J. Wang, J. S. Kurtz, Y. Liu, and M. H. W. Chan, Phys. Rev. B 71, 104521 (2005).

${ }^{34}$ D. G. Naugle, J. W. Baker, and R. E. Allen, Phys. Rev. B 7, 3028 (1973).

${ }^{35}$ W. A. Little and R. D. Parks, Phys. Rev. Lett. 9, 9 (1962).

${ }^{36}$ R. Prozorov, A. F. Fidler, J. Hoberg, and P. C. Canfield, Nat. Phys. 4, 327 (2008). 